\title{
Forest Fire Detection System
}

\author{
D.Sathya
}

\begin{abstract}
The forest is one of the most important wealth of every country. The forest fires destroys the wildlife habitat, damages the environment, affects the climate, spoils the biological properties of the soil, etc. So the forest fire detection is a major issue in the present decade. At the same time the forest fire have to be detected as fast as possible. In the proposed method, a color spatial segmentation, temporal segmentation, global motion compensation, Support Vector Machine (SVM) classifications are used to detect the fire and to segment the fire from the video sequence. The method is implemented over the two real time data sets. The proposed method is most suitable for segmenting fire events over unconstrained videos in real time.
\end{abstract}

Keywords: Fire-like pixel detector, Regions of interest, Support Vector Machine, Temporal Segmentation.

\section{INTRODUCTION}

Forest is one of the major wealth of our country. Forests provide enormous material goods and environmental services. They are useful for industry as well as rural economic growth. Forests provide timber, resins, rubber, food items, medicines etc. Forests also provide best environmental service to the world. The trees in the forests produce oxygen by photosynthesis, which reduces global warming. Forests absorb carbon dioxide which is a raw material for photosynthesis. Forests prevents soil erosion, absorb toxic gases. Forests are the homes for wild animals. At the same time, when the forest is under fire it emits lot of carbon dioxide leads to climate change and global warming. So the forest fire has to be detected at earlier stage. Due to isolation, inaccessibility, tough weather, shortage of frontier staff, the early finding of forest fire is a difficult task.

Nowadays, the vision based fire detection method replaces the conventional fire detection methods. The digital camera and content based video processing technologies are used to implement the vision based fire detection. The characteristic feature like fire, motion and geometry are used in vision based fire detection systems.

They uses color information as a preliminary processing step for detecting fire. In [1], the variation of temporal values from a small subset of images and the color predicate information are used to identify fire from video sequences. The training set uses a physically segmented fire set to recognize the color pixels in a fire. In [2], chromatic features and some dynamic features are used to extract the fire from video sequences.

In [3], the flames are detected by taking the input from video sequence captured by a camera. The hidden Markov model is applied to detect the flame flicker process. The system uses the following fire detection algorithm: i) It determines the regions in the video frame or moving pixels, ii) The color in the moving pixel are matched with the flame color, if color match is found then hidden Markov model is applied temporarily and spatially.

In [4], proposed two methods to detect fire from continuous video sequence. i) the background subtraction method is used for identifying fire pixels from the background image, ii) the statistical model is used for classifying the fire pixels. In [5], YCbCr color space is taken to distinguish the luminance from chrominance information. The rule based approach is replaced by the fuzzy logic approach for detecting fire pixels. In addition, the statistically derived chrominance model is used to discriminate fire and non fire pixels.

In the present work, the following methods are used to achieve better fire detection accuracy and segmentation of fire from real time videos. The proposed work implements the color spatial segmentation, temporal segmentation, global motion compensation and temporal classification methods. The paper is organized as follows: Section 2 describes the need for the system, Section 3 explains about the proposed system, Section 4 describes the prototype model and its results, and Section 5 explains the conclusion and future work

\section{II.REQUIREMENT OF THE SYSTEM}

Existing systems uses electronic sensors to detect fire or smoke. The change in temperature indicates the presence of fire or smoke in a region which can be detected by the sensors using radiation heat. As forests are in a remote location, installation and maintenance of sensors over large area is difficult. So the sensors cannot be used to deploy over large area such as forests, petrochemical plant, and saw mills etc [10]. The other consequence is, the sensor would detect heat or smoke only when it reaches nearer.

Nowadays, the vision based fire detection technique is used widely to detect fires. Along with the surveillance systems the vision based fire detection technique can be incorporated at relatively low additional cost. The advantages of vision based fire detection techniques are listed here:i) the fast response to fires. ii) the location of fire is sensed using this method not just the radiation, iii) the captured images can be analyzed and it can be used for future purposes and storage, iv) it can be used for outdoor places which covers large area.

\section{III.PROPOSED SYSTEM}

The presences of fire in video streams are indicated by semantic events. Most of the existing systems can only be used for the videos obtained from stationary cameras and videos obtained from the controlled lightening conditions. These existing automatic fire detection systems cannot be 
used for video streams obtained from mobile phones or any hand held devices. The KILLFIRE method is proposed to overcome these limitations. The KILLFIRE method works on three sections: i) To improve the accuracy, the Fire-like pixel detector color model is used, ii) To avoid the problem occurring in stationary videos, the new technique of motion compensation is used, iii) To identify and segment the fire in video streams, the segmentation method is used. The KILLFIRE is implemented over the two video set with different characteristics. The components of KILLFIRE method are

-A color spatial segmentation is used to pre-select the region that is expected as fire pixels.

-The extraction of sparse flow in the fire region and extraction of dense flow in the background is done by temporal segmentation.
-A global motion compensation using a block-based motion estimation

-A SVM classification uses experimental motion patterns to classify the region is fire or not.

\section{A.Color spatial segmentation}

The color based modelling for detecting fire is a good approach, because it performs faster than any other traditional strategies. The color based segmentation reduces the content before performing any complex calculations. The proposed color model is used to detect fire on videos. The Fire-like Pixel Detector (FPD)perform the spatial segmentation as an preliminary step of the fire detection

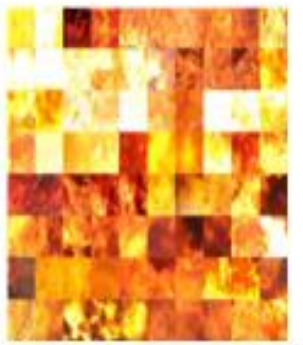

(a)

(b)

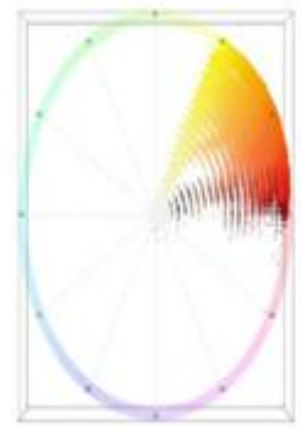

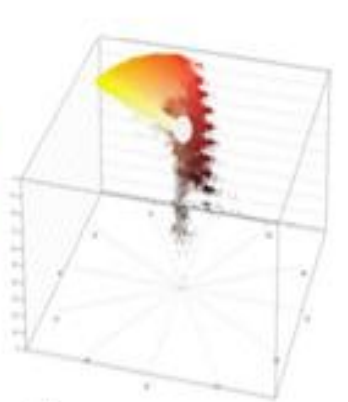

(c)

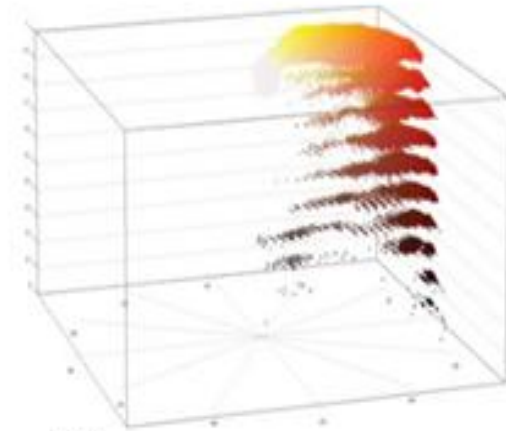

(d)

Figure 1: Fire color pixels visualization on HSV color space

The proposed model analyse the region of interest (ROI's) from the fire images. For analysing the ROI's the 3D cylindrical histogram is used to examine the relationship among three HSV components. The fire color pixel visualization is shown in Fig.1. The Fig.1(a) shows ROI's in fire image, Fig.1 (b) shows the fire color pixel distribution which highlights the saturation and hue components. When the hue component is around 00 (red), then the saturation will have more intensity. If a hue is around 600(yellow), then the saturation will have lesser intensity and evenly distributed. Fig. 1(c) and 1(d) shows two complementary points of view of the HSV distribution. If the value point is high then the hue is close to yellow.

\section{B.Temporal segmentation}

The KILLFIRE method analyses the motion pattern of fire region after performing the color spatial segmentation using FPD. It and If is created, the two image matrices for sparse optical flow estimation and dense optical flow estimation. The following equations are used to compute these two matrices.

The figure 2 gives the overview of KILLFIRE method which consists of 4 modules: A) The spatial segmentation by means of the FPD model. B) The temporal segmentation with the estimated flow in the candidate region and the background [7]. C) Estimation of the global flow and motion compensation. D) SVM Classification for the motion pattern.

The disparity existing in equation confines the $\mathrm{H}$ component to the interval of yellow-red dominant colors. The equation, in turn, confines the minimum values for $\mathrm{S}$ and $\mathrm{V}$ as a function of component $\mathrm{H}$. Given a fixed $\mathrm{H}$ in the precise range, the $\mathrm{S}$ and $\mathrm{V}$ components are limited by a circumference centered at $(1,1)$. The radius varies according to component $\mathrm{H}$. The radius of this circle has maximum value when $\mathrm{H}=0$ (pure red) and decreases quadratically.

\section{C.Motion estimation}

The motion estimation can be done after the computation of It and ItF. The motion estimation is possible by using sparse motion estimation, and dense motion estimation [8].

Sparse motion estimation: The processing time can be reduced by first detecting points of interest to track in the candidate region instead of processing all the pixels in a segment. The Harris Corner detector is used to extract the points of interest. 


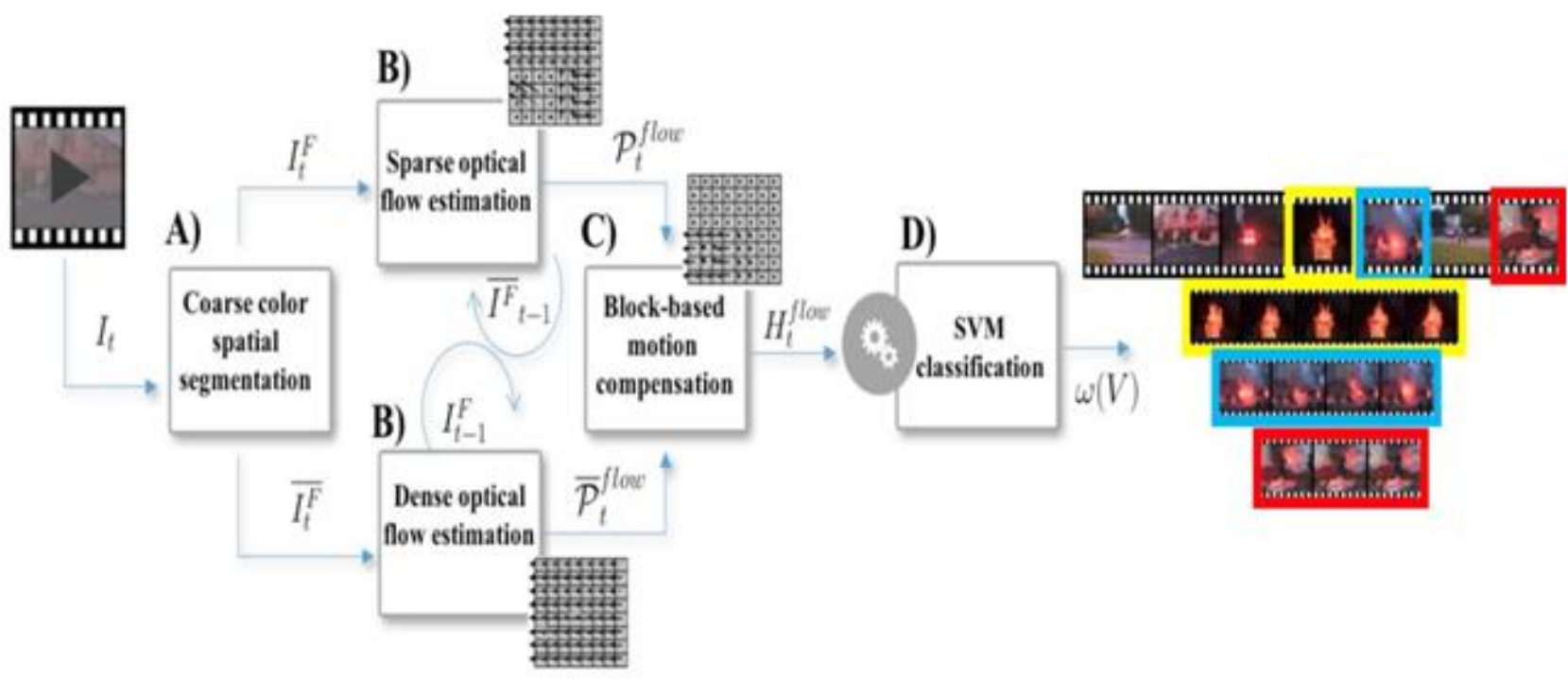

Figure 2 Overview of the KILLFIRE method

\section{Dense motion estimation:}

The sparse optical flow selects the dynamic information that is more related with target of interest for the fixed camera. When there is a camera motion, the resulting movement suppresses the object motion information. The frame ItF represents the background of the scene can be used to extract the dense optical flow information. The tracking of dense information is used to extract the global motion from the entire scene. The dense motion estimation considers all the points in the flow field and the points are sampled at uniform intervals. The Gunnar Farneback's algorithm is used to generate the flow dense flow Pt.

\section{Block-based motion compensation:}

Sparse motion estimation and dense motion estimation are used to extract the patterns from stationary cameras. These estimations cannot be used for moving cameras as in the cell phone. Due to the movement of cameras, the extra motion component will be added to every frame. The block based motion compensation partition the frames into smaller blocks. The global flow estimation is calculated from these blocks. After calculation, the flow component is subtracted from the original frame.

From the ItF, the dense motion is extracted and is used for block-based motion compensation to identify camera motion. The mean local flows are used to estimate the global flow. To calculate the local flow, the frame ItF, is divided into 32 × 32 pixels of non-overlapping blocks. The mean direction is calculated for each block bit and is also called as block dominant flow pit. To do this, the direction of the flow is to be calculated. So, the flow field is divided into four quadrant sets to calculate the direction of flow form updown and left-right directions.

Then the direction flows are grouped into their quadrants. Let $\mathrm{Qs}=\{\varphi \mathrm{k}: 1 \leq \mathrm{k} \leq \mathrm{ns}\}$, where $\mathrm{ns}=|\mathrm{Qs}|$, be the set hold the orientation flows of the quadrant with the highest amount of flow. After the calculation of block dominant orientation pit of all the blocks, the block dominant flows are quantized into global histogram of the background flow
HtG. The global histograms are sampled into eight bins of equal width.

\section{D.Support Vector Machine classification}

The SVM is used in almost all the applications particularly for event detection [11]. From the calculated histograms, the heat flow of each frame is given as input for SVM classifier. The SVM classifies whether the given motion pattern is fire transition or not. The real time dataset is used to implement the SVM, for which $30 \%$ of the fire videos is taken for training phase and the remaining $70 \%$ of the videos is taken for testing phase.

\section{IV.PROTOTYPE\& RESULTS}

The fig. 3 and fig. 4 shows the prototype and the conversion models. The modules work independently of each other. Each modules converts video into frames and each frame is converted to bitmap image. Here the three clones of the bitmap is taken and converted into $\mathrm{Y}, \mathrm{Cb}, \mathrm{Cr}$ channels [9].

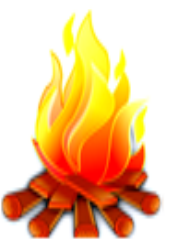

Fire Location

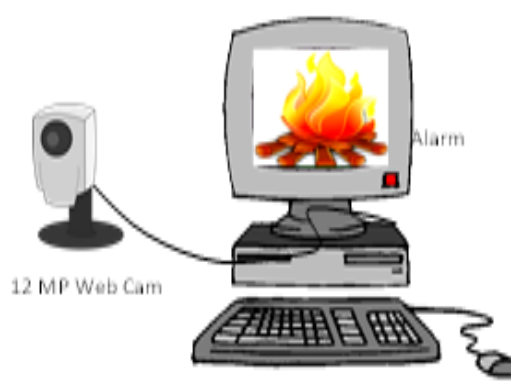

Computer
Figure 3 Prototype Model 

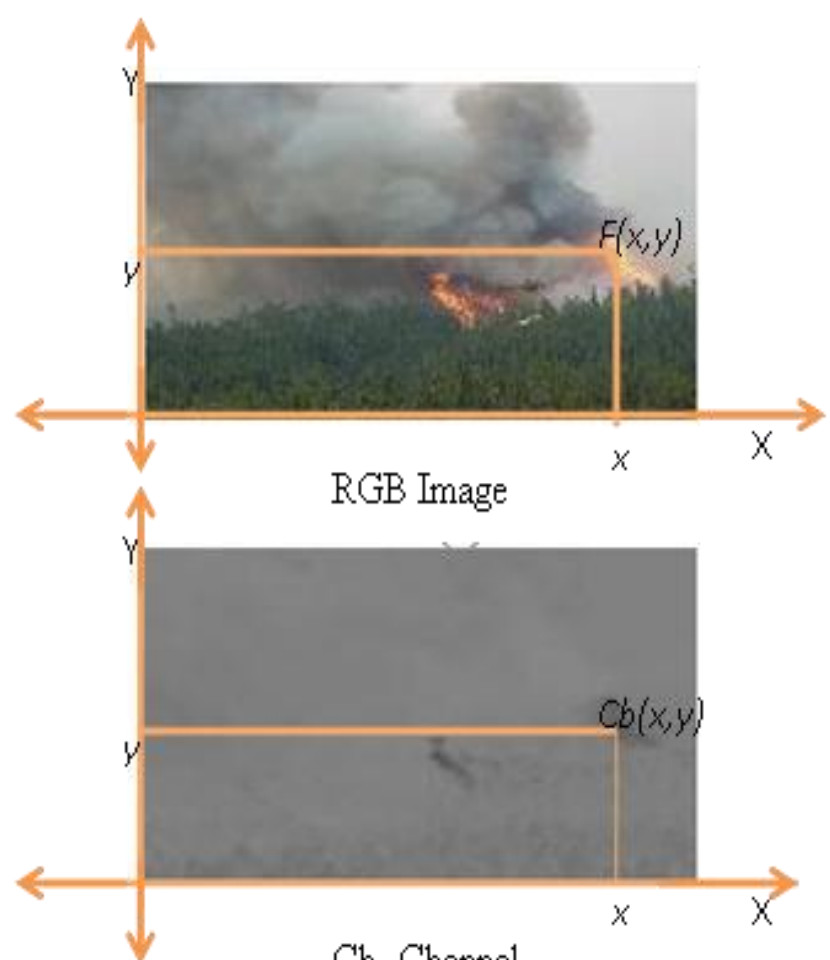

$\mathrm{Cb}$ Channel

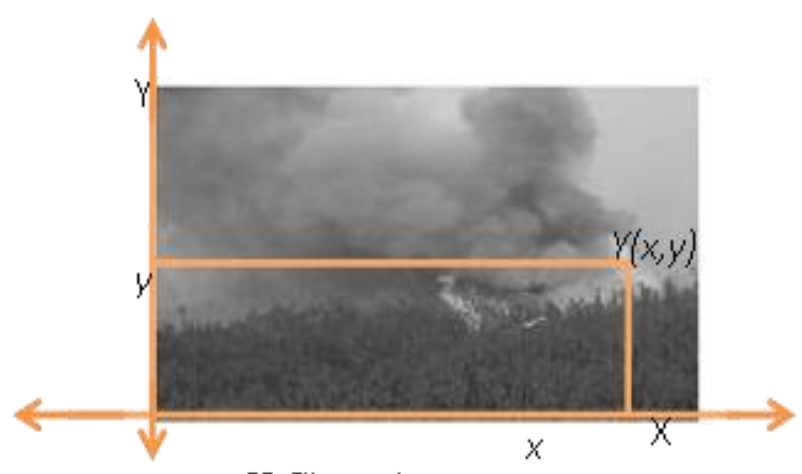

Y Channel

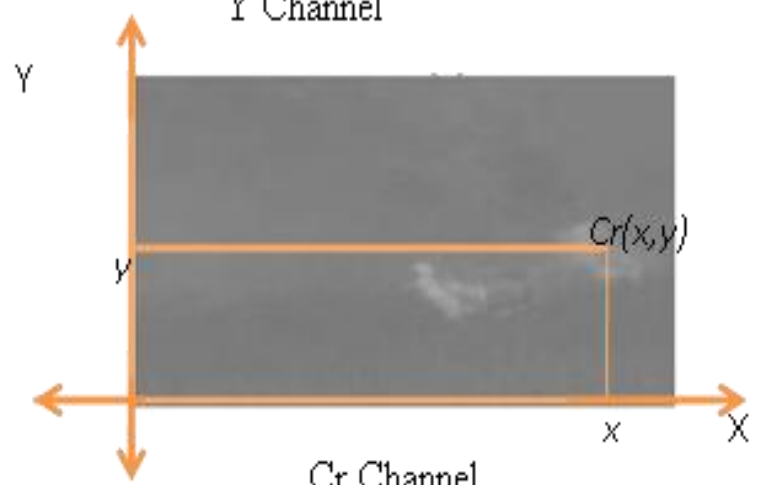

Figure 4 Conversion Models

$\mathrm{Y}=0.299 \mathrm{R}+0.587 \mathrm{G}+0.114 \mathrm{~B}$

$\mathrm{Cb}=-0.169 \mathrm{R}-0.331 \mathrm{G}+0.500 \mathrm{~B}+128$

$\mathrm{Cr}=0.500 \mathrm{R}-0.419 \mathrm{G}-0.082 \mathrm{~B}-128$
The difference of $\mathrm{Y}-\mathrm{Cb}$ and $\mathrm{Cr}-\mathrm{Cbparameters} \mathrm{is} \mathrm{taken} \mathrm{as}$ fire pixel. The conversion model is shown in figure 4.The results are shown in the figure 5 and 6.

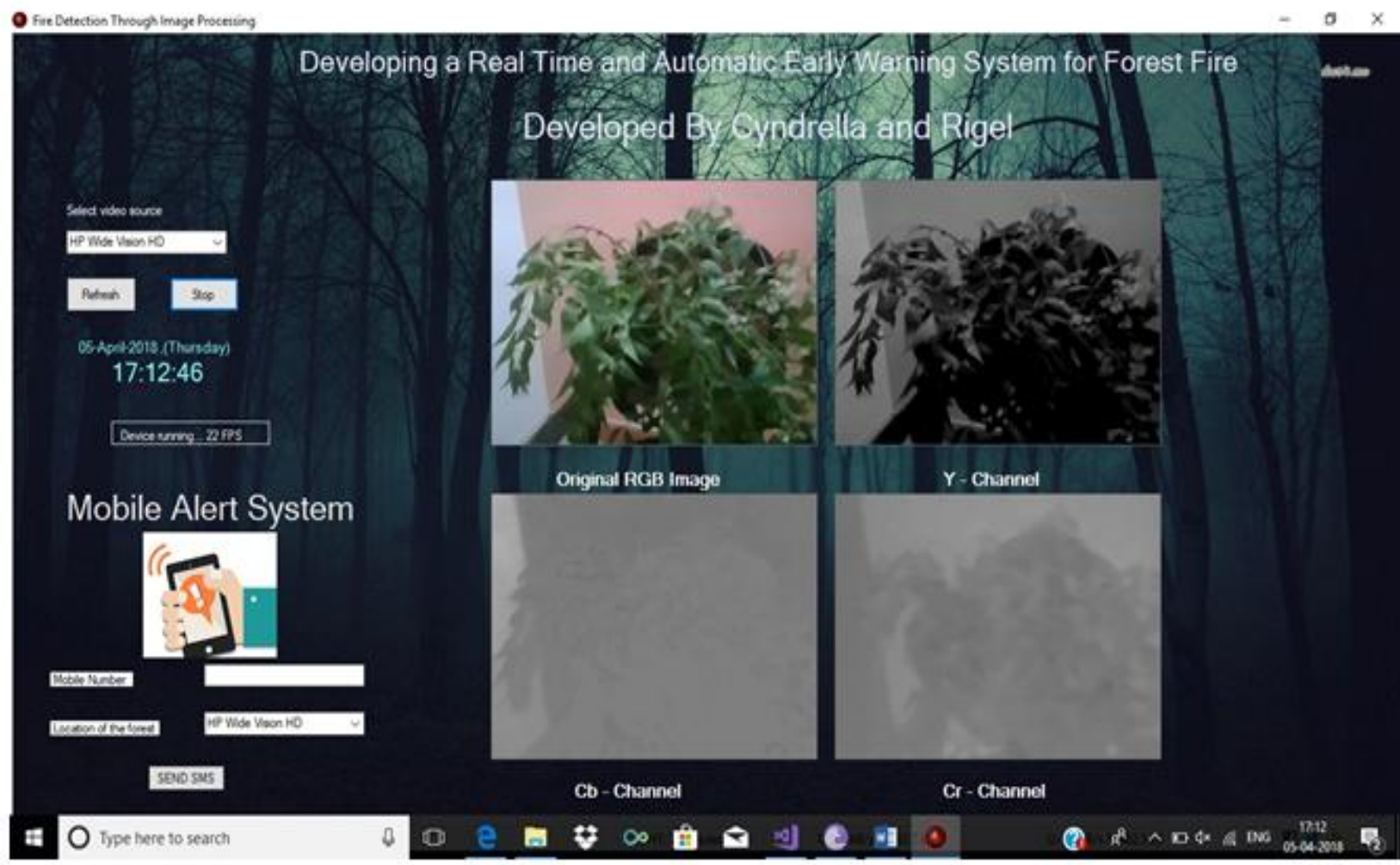

Figure 5 Screenshot for Extraction of Image Components

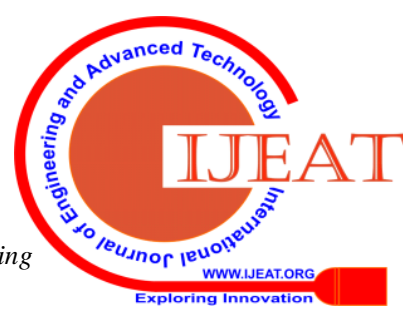




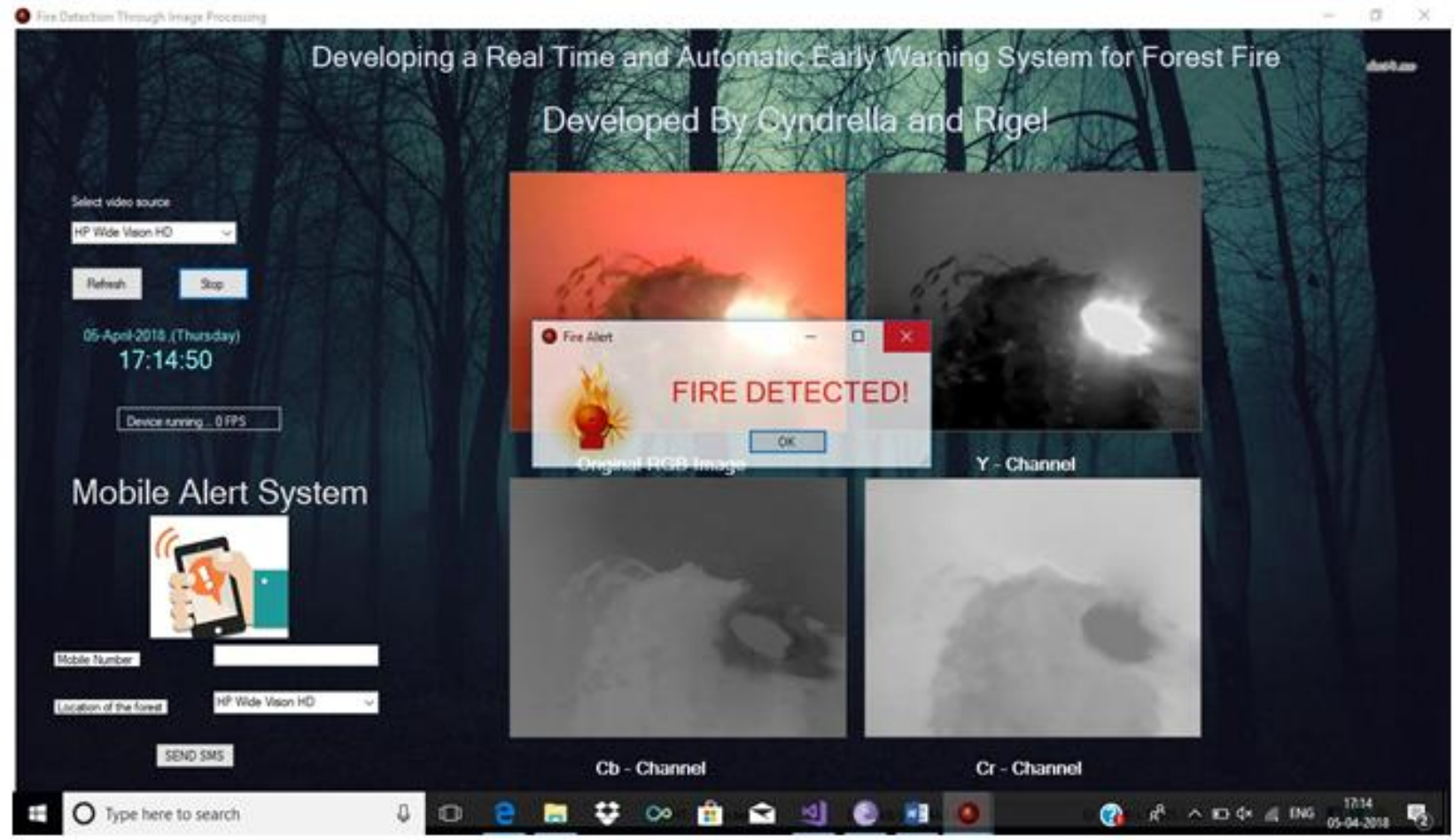

Figure 6 Screen shot of Fire Detection

\section{V.CONCLUSION AND FUTURE WORK}

The proposed KILLFIRE method uses spatial segmentation and motion flow estimations to detect fire from moving videos.By the use of motion compensation techniques, high accuracy is achieved in the results. The temporal video segmentation adds the extra advantage in the proposed system which segments the fire regions.

The proposed system is implemented with the real time datasets which signifies that the KILLFIRE method is more appropriate for real-time unconstrained motion videos.

\section{REFERENCES}

1. W.P.III, M.Shah, and N.da Vitoria Lobo (2002) 'Flame recognition in video', Pattern Recognition Letters, Vol. 23, No. 13, pp. 319 - 327, 2002.

2. T.H.Chen, P.H.Wu, and Y.C.Chiou (2004) 'An early firedetection method based on image processing', ICIP, Vol. 3, pp. 1707-1710.

3. B.U. Toreyin, Y. Dedeoglu, and A.E. Cetin (2005) 'Flame Detection in Video Using Hidden Markov Models',Proc. IEEE Int. Conf. Image Process., pp. 669672 .

4. T. Celik et al. (2007) 'Fire Detection Using Statistical Color Model in Video Sequences', J. Visual Commun. Image Representation, Vol. 18, No. 2, pp. 176-185.

5. T. Celik, H. Demirel, and H. Ozkaramanli, (2007) 'Fire Pixel Classification Using Fuzzy Logic And Statistical Color Model', IEEE International Conference on inAcoustics, Speech, and Signal Processing, USA.

6. Letricia P. S. Avalhais, Jose Rodrigues-Jr., Agma J. M. Traina (2016) 'Fire detection on unconstrained videos using color-aware spatial modeling and motion flow', IEEE 28th International Conference on Tools with Artificial Intelligence.

7. P.Barmpoutis, K.Dimitropoulos, and N.Grammalidis (2013) 'Real time video fire detection using spatiotemporal consistency energy', IEEE AVSS, pp. 365-370.

8. Ms. Bhavina Patel, Dr.R.V.Kshirsagar, Dr.Vilas Nitnaware (2013) 'Review And Comparative Study Of Motion Estimation Techniques To Reduce Complexity In Video Compression', International Journal of Advanced Research in Electrical, Electronics and Instrumentation Engineering, Vol. 2, Issue 8.

9. Jonas Gomes, Luiz Velho (1997) 'Image Processing for Computer Graphics', Springer.

10. Nagaraj, B., and P. Vijayakumar. "Controller tuning for industrial process-a soft computing approach." Int. J. Advance. Soft Comput. Appl 4, no. 2 (2012).

11. Sathya.D, Krishneswari.K (2016) 'Cross Layer Intrusion Detection System for Wireless Sensor Networks', Journal of Scientific and Industrial Research, Vol.75, pp.213 220.

12. S.Pradeepa, Yamunarani S, D Sathya,(2015) 'Intelligent Intrusion Detection System using Hybrid Techniques in Wireless Sensor Networks', International Journal of Advance Research In Science And Engineering, Vol.4, No. 1, pp. 390- 398.

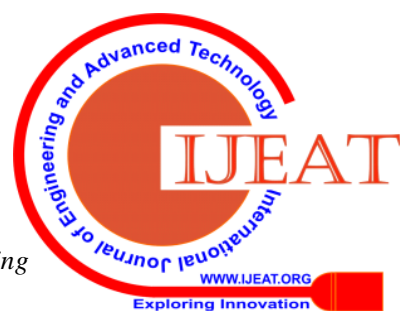

